\title{
A Novel Electrocardiographic Sign of an ST-Segment Elevation Myocardial Infarction-Equivalent: De Winter Syndrome
}

\author{
Mustafa Ugur Goktas ${ }^{\mathrm{a}}$, Ozgur Sogut ${ }^{\mathrm{a}, \mathrm{b}}$, Mehmet Yigit ${ }^{\mathrm{a}}$, Onur Kaplan ${ }^{\mathrm{a}}$
}

\begin{abstract}
Patients with de Winter syndrome, also termed anterior ST-segment elevation myocardial infarction (STEMI)-equivalent, represent $2 \%$ of all patients with acute anterior myocardial infarctions admitted to emergency departments (EDs). STEMI-equivalents do not present with classical electrocardiogram (ECG) changes but exhibit a critical stenosis of the left anterior descending (LAD) coronary artery. This is under-recognized by clinicians and is therefore associated with high morbidity and mortality. Here, we report a rare case of a novel, typical, STEMI-equivalent ECG pattern without obvious ST-segment elevation in a 34-year-old female who presented to our ED with substantial chest pain and a large, acute, transmural anterior myocardial infarction caused by acute occlusion of the LAD coronary artery. However, she presented as a non-STEMI case. A definite diagnosis of de Winter syndrome was made on the basis of clinical and ECG findings.
\end{abstract}

Keywords: De Winter syndrome; STEMI-equivalent; LAD coronary artery; Anterior wall acute myocardial infarction; Revascularization

\section{Introduction}

De Winter syndrome is a distinct form of anterior ST-segment elevation myocardial infarction (STEMI)-equivalent, characterized by a unique electrocardiogram (ECG) pattern $[1,2]$. The key diagnostic features of the ECG pattern include an upsloping ST-segment depression $>1 \mathrm{~mm}$ at the $\mathrm{J}$ point in the precordial leads; tall, prominent, and symmetrically peaked T waves with no classic ST-segment elevation in the precordial leads; and slight ST-segment elevation $(0.5-1 \mathrm{~mm})$ in the aVR lead [3-5]. It has been suggested that the positive predictive value of this ECG pattern is $100 \%$, thus very strongly support-

Manuscript submitted July 15, 2017, accepted August 3, 2017

aDepartment of Emergency Medicine, Haseki Training and Research Hospital, University of Health Sciences, Istanbul, Turkey

${ }^{b}$ Corresponding Author: Ozgur Sogut, Department of Emergency Medicine, Haseki Research and Training Hospital, University of Health Sciences, Millet Street, 34096, Fatih/Istanbul, Turkey. Email: ozgur.sogut@sbu.edu.tr

doi: https://doi.org/10.14740/cr576w ing a clinical diagnosis of de Winter syndrome [3]. Patients with this syndrome have a critical obstruction in the left anterior descending (LAD) coronary artery, causing extensive anterior-wall myocardial infarction [5]. Recognition of the ECG pattern is of vital importance; such patients must be triaged for emergency reperfusion therapy combined with fibrinolysis or percutaneous coronary intervention (PCI) [4].

\section{Case Report}

A 34-year-old female with a history of heavy smoking and hypercholesterolemia presented to our emergency department (ED) with compressive chest pain 2 days in duration. On physical examination, she was conscious but extremely anxious. Her blood pressure was 120/80 mm Hg, her pulse rate was 81 beats/ $\mathrm{min}$, and her respiratory rate was 18 breaths/min (on a non-rebreather mask) with a pulse oximetric figure of $96 \%$. On cardiac examination, the S1 and S2 were normal and no heart murmur was apparent. The 12-lead ECG obtained at admission revealed significant ST-segment depression $(>1 \mathrm{~mm})$ at the $\mathrm{J}$ point in leads V3-V6, with tall, positively symmetrical $\mathrm{T}$ waves. Slight ST-segment elevation $(0.5 \mathrm{~mm})$ was evident in the aVR lead (Fig. 1). Such an electrocardiographic profile indicated that the patient had de Winter syndrome, an anterior STEMI-equivalent associated with acute occlusion of the LAD coronary artery and no ST-segment elevation in the precordial leads of the 12-lead ECG. Dual antiplatelet therapy with aspirin and a direct-acting P2Y12 receptor inhibitor (prasugrel) was commenced in the ED. Urgent, diagnostic coronary angiography revealed total occlusion of the middle of the LAD coronary artery (Fig. 2). PCI was performed and a $2.5 \times 18 \mathrm{~mm}$ drug-eluting stent was placed, after dilatation of the affected region using a $1.5 \times 12 \mathrm{~mm}$ balloon under $10 \mathrm{~atm}$ of pressure (Fig. 2a). This was successful; arterial blood flow was re-established (Fig. 3). The ECG returned to normal (Fig. 4). As no complication developed in hospital, the patient was discharged free of angina 3 days later. We scheduled smoking-cessation and lifestyle counseling.

\section{Discussion}

This case demonstrates the importance of immediate mechanical reperfusion therapy for a patient with de Winter syndrome, 


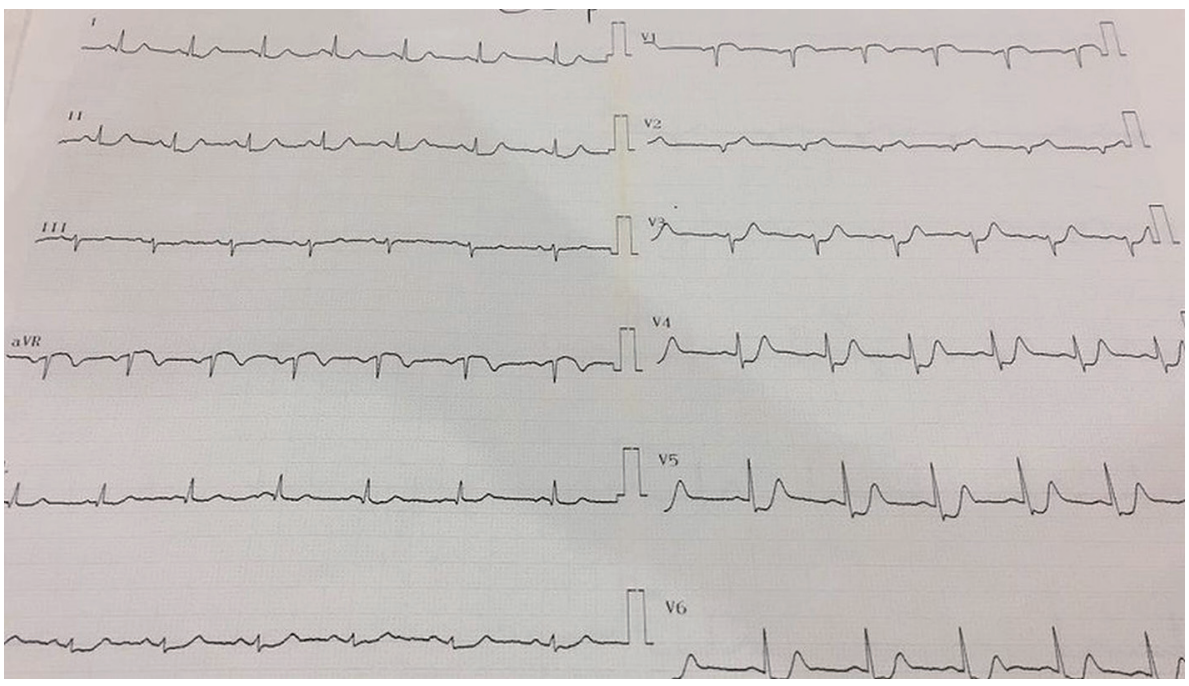

Figure 1. The 12-lead electrocardiogram (ECG) taken at the time of admission to the emergency department revealed upsloping ST-segment depression at the $\mathrm{J}$ point in leads V3-V6, with prominent T waves and slight ST-segment elevation evident in the aVR lead.

which is associated with prominent chest pain and a characteristic ECG pattern lacking the classic ST-segment elevation. The de Winter ECG pattern is similar to that of an anterior STEMI, thus mimicking non-ST-elevation acute coronary syndrome [6]. Patients with the de Winter ECG pattern are younger, more commonly male, and have a higher incidence of dyslipidemia compared with patients exhibiting LAD coronary artery occlusion and classical STEMI on ECGs [7].

Data on the ECG pattern associated with de Winter syndrome are limited; 71 cases have been reported to date $[1,3-6$,

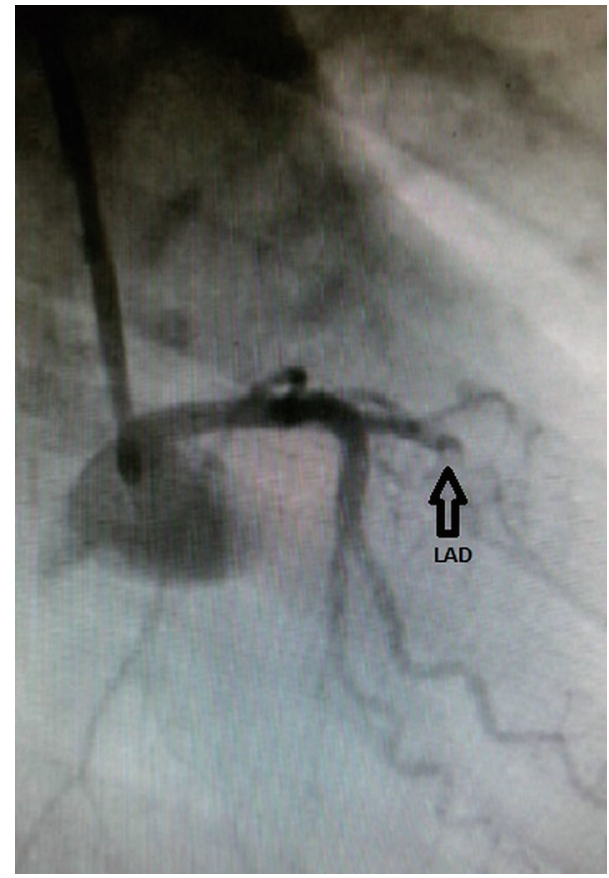

Figure 2. A left coronary angiogram revealing a complete mid-left anterior descending (LAD) coronary artery occlusion (arrow).
8]. The two largest series were retrospective analyses of 65 patients [1-3]; the others were sporadic cases $[3,4,6,8]$. The de Winter ECG pattern was first described in the 2008 case series of de Winter et al, who recognized the characteristic ECG pattern in a primary PCI database. A total of 30 of 1,532 patients with anterior myocardial infarctions caused by LAD coronary artery occlusions ( $2 \%$ of all cases) exhibited the characteristic ECG pattern [1]. Verouden et al documented the same ECG pattern in a 2009 case series; 35 of 1,890 patients $(2 \%)$ requiring PCI of the LAD coronary artery exhibited the pattern. De

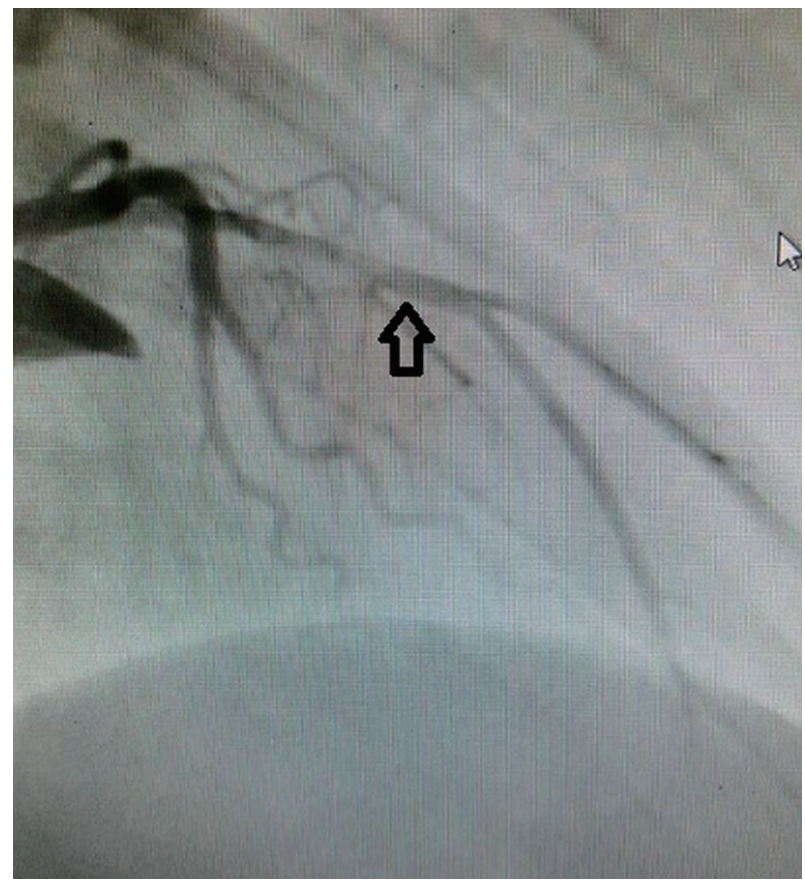

Figure 3. Blood flow was restored after percutaneous coronary intervention of the LAD coronary artery (arrow). 


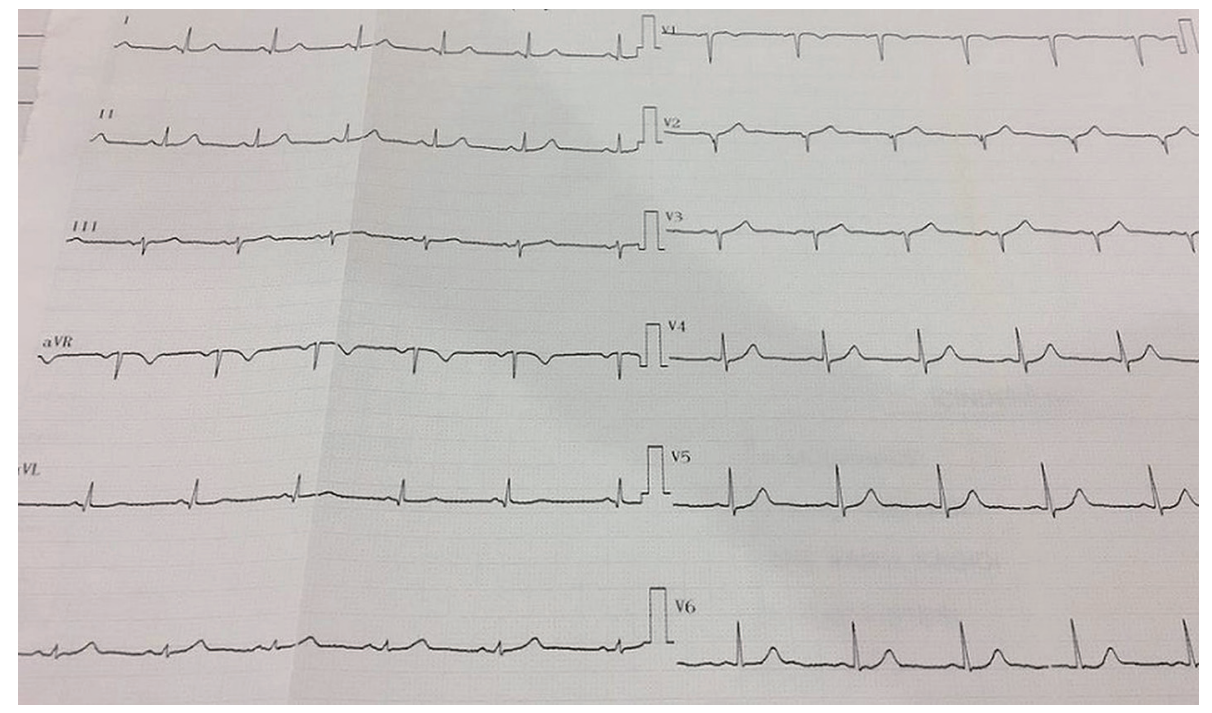

Figure 4. An ECG taken after stent placement, showing complete resolution of the ST-T waves.

Winter et al also reported three cases with typical symptoms of acute myocardial infarction accompanied by ECG patterns lacking ST-segment elevation and exhibiting slight J-point ST wave depression combined with high symmetrical T-waves. Urgent coronary angiography revealed complete occlusion of the LAD coronary artery in these patients, who were successfully treated via mechanical reperfusion therapy and stenting [4]. Martinez-Losas and Fernandez-Jimenez reported a case of de Winter syndrome associated with acute occlusion of the midLAD coronary artery in an elderly male smoker with hypercholesterolemia, which was treated via angioplasty and drug-eluting stent placement [5]. Our present case exhibited the typical chest pain and the ECG suggested complete occlusion of the midLAD coronary artery (ST elevation in the aVR lead of $0.5 \mathrm{~mm}$ and the absence of overt ST-elevation, with prominent T-waves, in the precordial leads). The ECG taken after successful primary PCI revealed complete resolution of the ST-T waves. The current 2013 American College of Cardiology Foundation/American Heart Association guidelines recommend that primary PCI within $2 \mathrm{~h}$ of first medical contact is the preferred reperfusion option in patients with the de Winter ECG pattern [9].

In conclusion, in patients presenting with anginal syndrome, ST-segment depression and peaked T waves, combined with the absence of classic ST-segment elevation in the precordial leads of the 12-lead ECG, suggest that a significant LAD coronary artery occlusion is present. It is of utmost importance to identify this distinct ECG pattern; timely investigation and revascularization of the LAD coronary artery are then possible. Early recognition of the STEMI-equivalent ECG pattern in patients with de Winter syndrome, followed by appropriate emergency reperfusion therapy (primary PCI), improves the clinical outcomes of these patients.

\section{Conflicts of Interest}

The authors have no conflicts of interest to disclose.

\section{References}

1. de Winter RJ, Verouden NJ, Wellens HJ, Wilde AA, Interventional Cardiology Group of the Academic Medical C. A new ECG sign of proximal LAD occlusion. N Engl J Med. 2008;359(19):2071-2073.

2. Gorgels AP. ST-elevation and non-ST-elevation acute coronary syndromes: should the guidelines be changed? J Electrocardiol. 2013;46(4):318-323.

3. Verouden NJ, Koch KT, Peters RJ, Henriques JP, Baan J, van der Schaaf RJ, Vis MM, et al. Persistent precordial "hyperacute" T-waves signify proximal left anterior descending artery occlusion. Heart. 2009;95(20):17011706.

4. de Winter RW, Adams R, Verouden NJ, de Winter RJ. Precordial junctional ST-segment depression with tall symmetric T-waves signifying proximal LAD occlusion, case reports of STEMI equivalence. J Electrocardiol. 2016;49(1):76-80.

5. Martinez-Losas P, Fernandez-Jimenez R. de Winter syndrome. CMAJ. 2016;188(7):528.

6. Goyal KK, Rajasekharan S, Muneer K, Sajeev CG. de winter sign: A masquerading electrocardiogram in ST-elevation myocardial infarction. Heart India. 2017;5(1):4850.

7. Rokos IC, French WJ, Mattu A, Nichol G, Farkouh ME, Reiffel J, Stone GW. Appropriate cardiac cath lab activation: optimizing electrocardiogram interpretation and clinical decision-making for acute ST-elevation myocardial infarction. Am Heart J. 2010;160(6):995-1003, e1001-1008.

8. Goebel M, Bledsoe J, Orford JL, Mattu A, Brady WJ. A new ST-segment elevation myocardial infarction equivalent pattern? Prominent T wave and J-point depression in the precordial leads associated with ST-segment elevation in lead aVr. Am J Emerg Med. 2014;32(3):287 e285-288.

9. O'Gara PT, Kushner FG, Ascheim DD, Casey DE Jr, 
Chung MK, de Lemos JA, Ettinger SM, et al. 2013 ACCF/AHA guideline for the management of ST-elevation myocardial infarction: a report of the American
College of Cardiology Foundation/American Heart Association Task Force on Practice Guidelines. Circulation. 2013;127(4):e362-425. 